\title{
Novel Active Bouncer Topology for Klystron Modulators based on Pulsed Transformers
}

\author{
Francisco Cabaleiro Magallanes, Davide Aguglia \\ CERN, Geneva, Switzerland, \\ Philippe Viarouge, Jérôme Cros \\ LEEPCI Lab, Québec, Canada
}

Keywords: «Pulsed Power», «Pulsed power converter», «Accelerators», «IGBT», «MOSFET».

\begin{abstract}
Active droop compensation systems, so called active bouncers, for klystron modulators based on monolithic pulse transformers perform the regulation of the output pulse voltage while simultaneously withstand all the primary current of the modulator. This imposes the utilization of high power semiconductors which can produce high switching losses and degrade the overall system efficiency. In order to overcome this issue, this paper proposes a new active bouncer topology based on the parallel connection of two different power converters: the first one is in charge of handling the majority of the primary current at high efficiency, and the second one is used to fine tune the bouncer voltage via a high bandwidth converter rated at a fraction of the first parallel connected converter. Detailed comparison between a classical active bouncer and two variants of the proposed topology are presented and based on numerical simulations.
\end{abstract}

Presented at: EPE 2015, 7-10 September 2015, Geneva, Switzerland

Geneva, Switzerland

October, 2015 


\title{
Novel Active Bouncer Topology for Klystron Modulators based on Pulsed Transformers
}

\author{
Francisco Cabaleiro Magallanes ${ }^{1,2}$, Davide Aguglia ${ }^{1}$, Philippe Viarouge $^{2}$, and Jerôme Cros ${ }^{2}$ \\ ${ }^{1}$ CERN-EUROPEAN ORGANIZATION FOR NUCLEAR RESEARCH \\ 1217 Geneva 23, Switzerland \\ Tel.: $+41 /(0)-227678489$. \\ E-Mails: fcabalei@cern.ch,davide.aguglia@cern.ch \\ URL: http://www.cern.ch \\ ${ }^{2}$ LEEPCI Lab., Laval University, Electrical and Computer Eng. Dept. \\ GIK 7P4, Québec (QC), Canada \\ Tel.: +1/ 41865621317139 \\ E-Mail: philippe.viarouge@ulaval.ca \\ URL: http://leepci.gel.ulaval.ca
}

\section{Keywords}

«Pulsed Power», «Pulsed power converter», «Accelerators», «IGBT», «MOSFET».

\begin{abstract}
Active droop compensation systems, so called active bouncers, for klystron modulators based on monolithic pulse transformers perform the regulation of the output pulse voltage while simultaneously withstand all the primary current of the modulator. This imposes the utilization of high power semiconductors which can produce high switching losses and degrade the overall system efficiency. In order to overcome this issue, this paper proposes a new active bouncer topology based on the parallel connection of two different power converters: the first one is in charge of handling the majority of the primary current at high efficiency, and the second one is used to fine tune the bouncer voltage via a high bandwidth converter rated at a fraction of the first parallel connected converter. Detailed comparison between a classical active bouncer and two variants of the proposed topology are presented and based on numerical simulations.
\end{abstract}

\section{Introduction}

The need for klystron modulators with improved performances, mainly for new linear accelerators, is growing more and more nowadays. Several existing klystron modulator topologies have been explored in the literature $[1,2,3,4,5]$; however, the vast majority of operational ones is based on the controlled discharge of a pre-charged capacitor bank. Therefore one of the options to achieve a precise square flat-top consists in increasing the size of the main capacitor bank, such that it experiences a limited voltage droop during the discharge. This option becomes inadvisable for medium and long pulse klystron modulators, specially taking into account not only the cost, but also the size and safety related to the stored energy. As an alternative solution, droop compensation systems or "bouncers", able to compensate a fraction of the capacitor nominal voltage (voltage droop during the pulse), have been proposed and successfully used in practice.

One of the topologies retained as a candidate for the CLIC Drive-Beam klystron modulators at CERN is based on a monolithic pulse transformer, as illustrated in Figure 1. In order to produce the required $180 \mathrm{kV}$ pulse, the main capacitor bank $C_{\text {main }}$ is pre-charged to a voltage of approximately $15 \mathrm{kV}$ and discharged through a step-up transformer by closing the main switch $S_{l} . C_{\text {main }}$ is dimensioned such it 
experiences a voltage droop of approximately $1 \mathrm{kV}$ during the pulse. In order to compensate this voltage

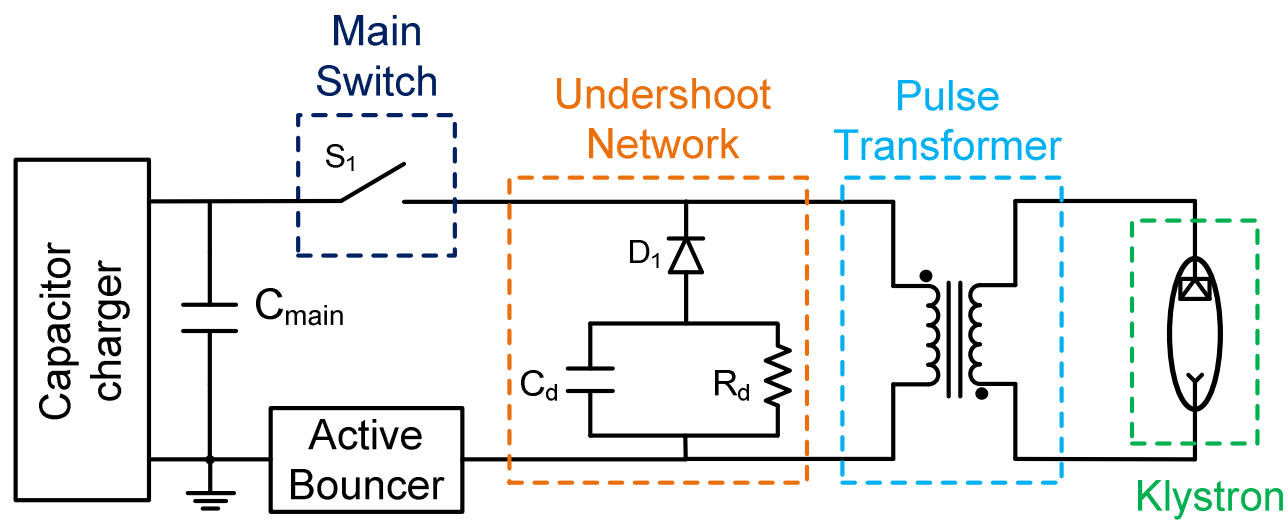

Fig. 1: Klystron modulator topology based on a monolithic pulse transformer.

droop, as well as the voltage across the transformer winding resistances and leakage inductances during the pulse (influenced also by the magnetizing current), an active bouncer circuit is installed in series with the main capacitor bank and the primary of the pulse transformer.

In this case a simple undershoot network guarantees a proper de-magnetization of the pulse transformer (which is equipped with a DC bias circuit for an inverse pre-magnetization action) between two consecutive pulses.

Table 1 shows the klystron modulator challenging specifications for the Compact Linear Collider (CLIC) [8], currently under study at CERN. We observe a rise time from $0 \mathrm{~V}$ to $180 \mathrm{kV}$ of only $3 \mu \mathrm{s}$, a settling time of $5 \mu \mathrm{s}$, and a flat-top stability of $0.85 \%$ for a $140 \mu \mathrm{s}$ flat-top length. The total complex will include several hundreds of these modulators synchronously pulsing and, therefore, modulator size, efficiency and cost must be optimized.

Table 1 CLIC Modulator's output pulse specification

\begin{tabular}{|l|c|r|}
\hline Nominal pulse voltage & $\mathrm{V}_{\mathrm{kly}}$ & $180 \mathrm{kV}$ \\
\hline Nominal pulse current & $\mathrm{I}_{\mathrm{sn}}$ & $160 \mathrm{~A}$ \\
\hline Pulse peak power & $\mathrm{P}_{\text {mod out }}$ & $28.8 \mathrm{MW}$ \\
\hline Rise \& fall times & $\mathrm{t}_{\text {rise }} \mathrm{t}_{\text {fall }}$ & $3 \mu \mathrm{s}$ \\
\hline Settling time & $\mathrm{t}_{\text {set }}$ & $5 \mu \mathrm{s}$ \\
\hline Flat-top length & $\mathrm{t}_{\text {flat }}$ & $140 \mu \mathrm{s}$ \\
\hline Repetition rate & $\mathrm{RR}$ & $50 \mathrm{~Hz}$ \\
\hline Voltage overshoot & $\mathrm{V}_{\text {ovs }}$ & $1 \%$ \\
\hline Flat-Top Stability & FTS & $0.85 \%$ \\
\hline Repeatability & REP & $10-50 \mathrm{ppm}$ \\
\hline
\end{tabular}

\section{Active bouncer topology overview}

Neglecting some non-linearity, the klystron behaves as a resistive load. Therefore, the modulator ideally produces a perfect current pulse. A common practice in klystron modulators consists in placing the droop compensation system in series with the main capacitor bank [6], as shown in Figure 1. However, this configuration presents an issue in applications where the rise time of the pulse is short, as when the main switch closes all the modulator current will suddenly flow into the bouncer filter capacitor, discharging it as presented in [7]. The bouncer is not able to compensate this modulator current perturbation until the current in its output filter inductances rises to the same current value, as illustrated in Figure 2.A). This perturbation must be taken into account when dimensioning the active 
bouncer output filter. In [6] an active bouncer circuit with a diode connected in parallel to the output filter capacitor, as illustrated in Figure 2.B), was proposed to prevent its discharge to negative values.

A)
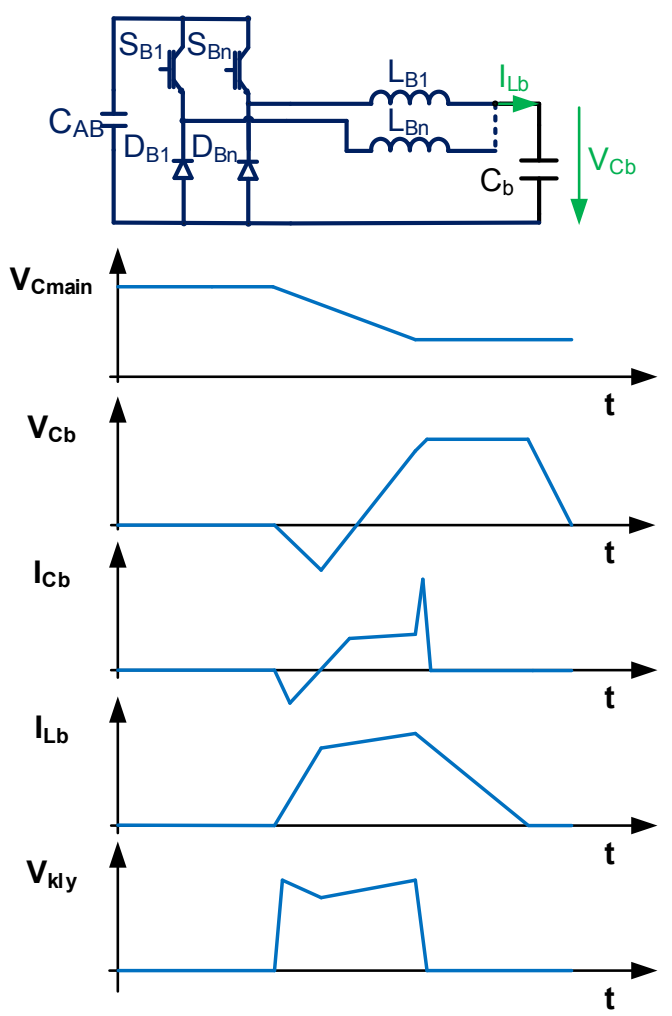

B)
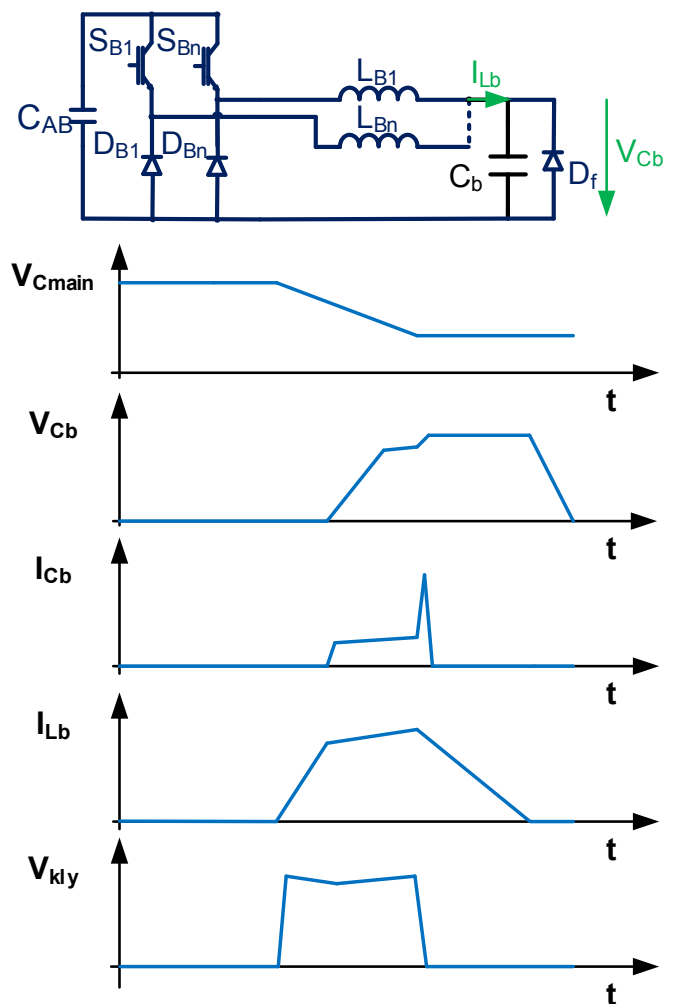

Fig. 2: Typical active bouncer topologies and associated simplified waveforms: A) Multiphase buck converter; B) Multiphase buck converter with blocking diode in parallel with the output filter capacitor.

However, in this configuration the active bouncer is still not able to charge the output filter capacitor to positive voltage values until its output current exceeds the modulator current. Therefore, this configuration is only suitable as a protection for long rise time modulator pulses. A different solution able to initialize the active bouncer output current and speed up the start of the droop compensation action in short rise time applications has to be addressed.

\section{Proposed active bouncer topology features}

In order to avoid the effects of the modulator current perturbation in short rise time applications previously introduced, the topology scheme of Figure 3 is proposed. Figure 4 illustrates the operation phases described hereafter:

1.-Current initialization: The switch $S_{S C}$ is closed and the active bouncer topology provides $\mathrm{V}_{\mathrm{AB}}$. The current $\mathrm{I}_{\mathrm{L}}$ rises.

2.-Pulse step-up: $S_{I}$ closes. $\mathrm{V}_{\text {kly }}$ rises and $\mathrm{I}_{\mathrm{L}}$ continues to increase.

3.-Flat-top: Once $\mathrm{V}_{\mathrm{kly}}$ achieves its nominal value, $S_{S C}$ is opened and the active bouncer topology is controlled to produce an output voltage ramp on $\mathrm{V}_{\text {bouncer }}$ to compensate for $C_{\text {main }}$ discharge.

4.-Recovery: $S_{l}$ is opened. The pulse transformer is demagnetized through the branch composed by $R_{d}, C_{d}$ and $D_{l} . \mathrm{V}_{\text {bouncer }}$ increases its value until it equals $\mathrm{V}_{\mathrm{AB}}$ voltage. Once this happens, the diode $D_{f}$ is forward-biased, so the energy stored in $L_{b}$ is recovered back to $C_{A B}$ passing through the diode $D_{f}$ and the free-wheeling diode(s) of the active bouncer topology.

5.-Reset: Once $\mathrm{I}_{\mathrm{L}}$ drops down to $0 \mathrm{~V}, C_{b}$ is discharged through $R_{R}$ by closing $S_{R}$. 


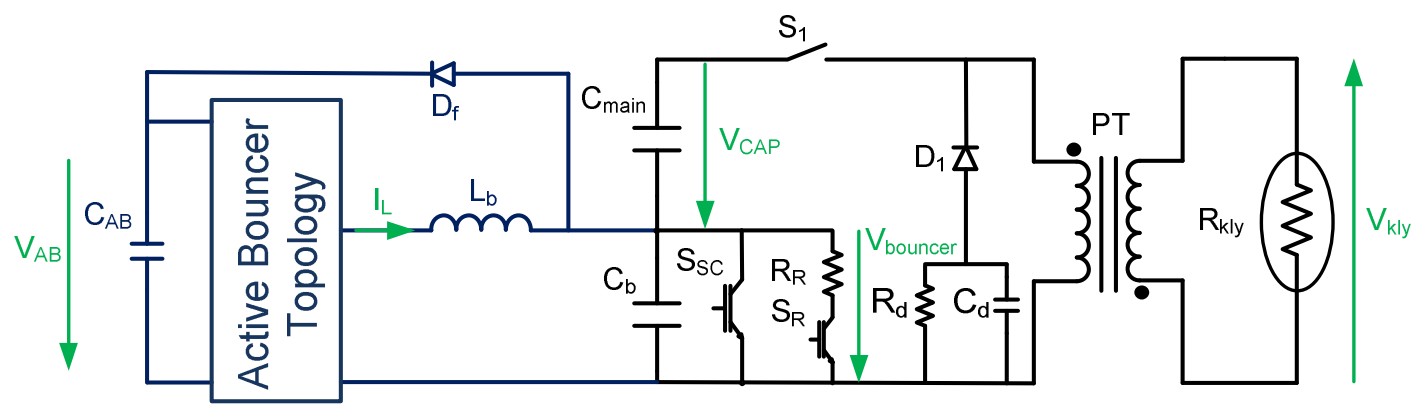

Fig. 3: Proposed topology scheme of the active bouncer.

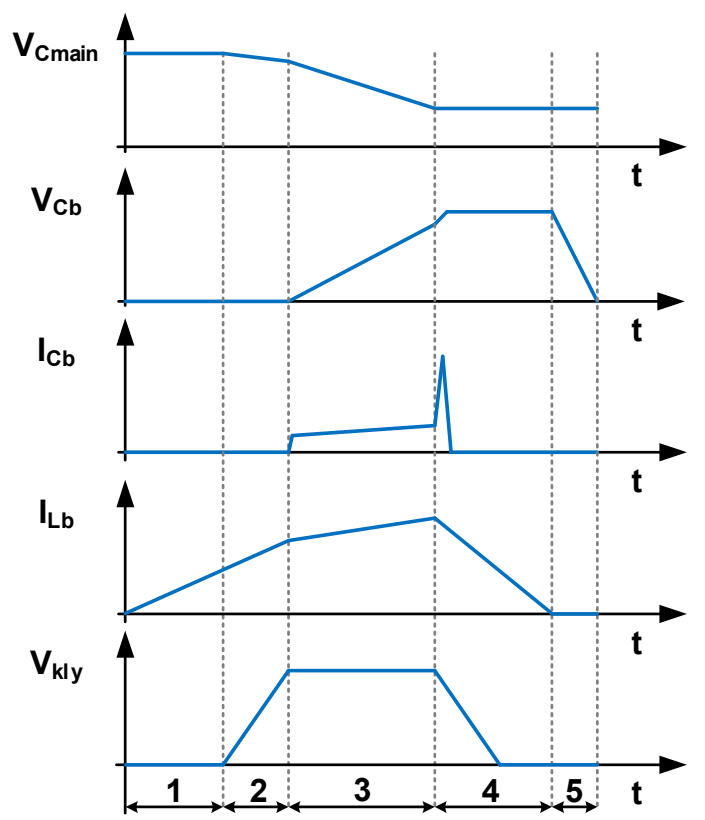

Fig. 4: Operational phases of the proposed topology.

Since $S_{S C}$ remains permanently closed during the pulse-step up, and neglecting the voltage drop across the switch, in order to achieve the nominal current value after the rise time, the current $\mathrm{I}_{\mathrm{L}}$ at the beginning of phase 3 must be:

$$
I_{L}(0)=I_{P}-t_{\text {rise }} \frac{V_{A B}}{L_{B}}
$$

where $I_{P}$ is the nominal primary current of the modulator. Therefore, the initialization of the current must be triggered at the time calculated in (2) before closing $S_{l}$.

$$
t_{\text {trig_init }}=\frac{L_{b}}{V_{A B}} I_{p}-t_{\text {rise }}
$$

\section{Proposed active bouncers topologies}

\section{Description of the proposed active bouncers}

Three topologies, illustrated in Figure 5, have been selected as candidates for the active bouncer. The first one (Figure 5.A) is based on a common multiphase buck-converter topology, as the one presented in [6]. The second and the third consist of a combination of power converters. They both share a common high-current and low switching-frequency stage, which manages all the modulator current. In 
addition, a second low-current high-bandwidth stage whose only task is to produce a voltage ramp on the bouncer output filter capacitance to compensate for the main capacitor voltage discharge, is connected in parallel. On the topology shown in Figure 5.B, this low-current stage consists of a high switching-frequency buck converter; whereas on the one shown in Figure 5.C, the low current-stage consists of a power MOSFET operated in its linear region.
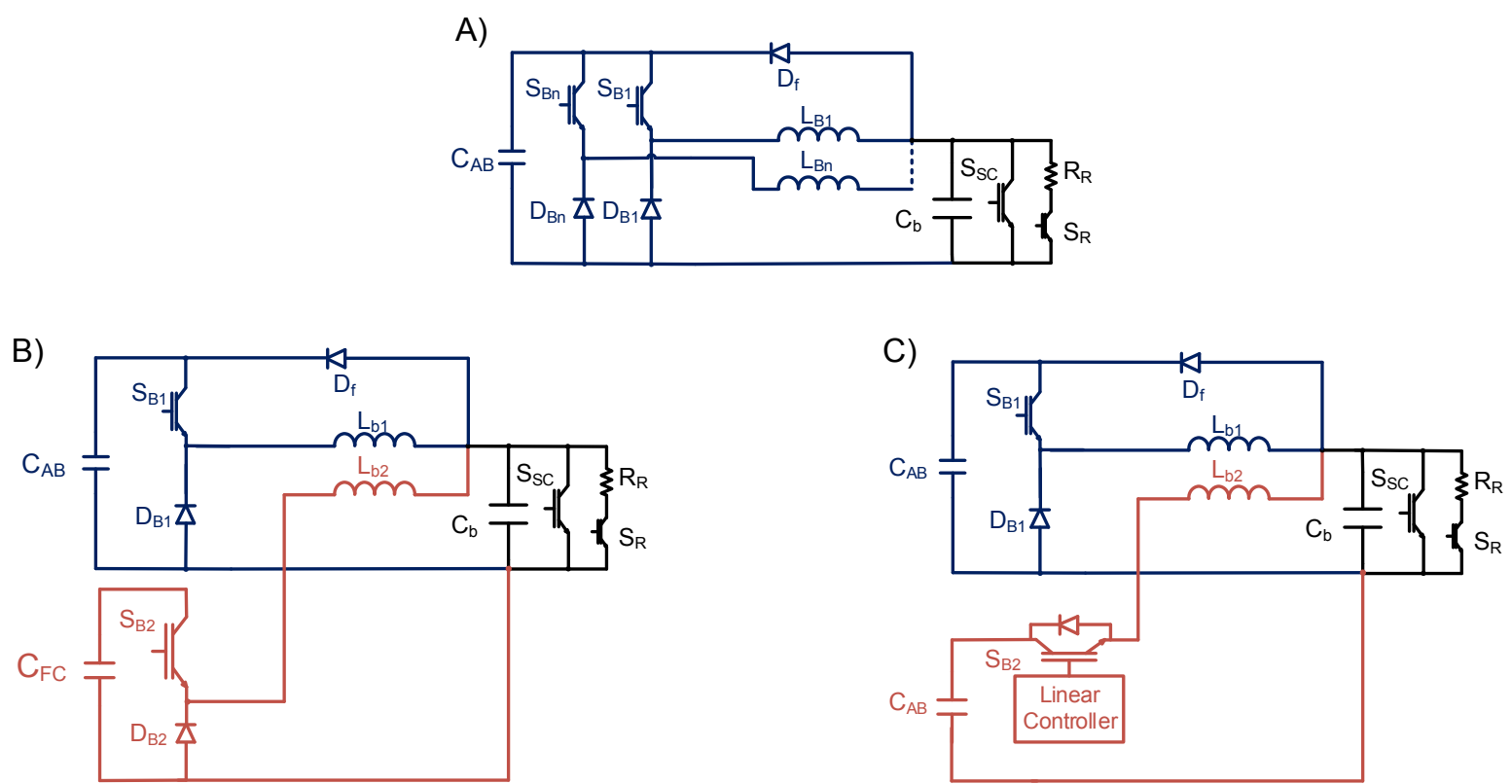

Fig. 5: Active bouncer topology candidates: A) Topology \#1: Multiphase buck converter; B) Topology \#2: Solution with high-current low switching-frequency buck stage combined with a low current high switchingfrequency buck stage; C) Topology \#3: Hybrid solution with high-current low switching-frequency buck stage and low current linear stage.

\section{Design and performances comparisons}

In order to evaluate their performances, the three topologies have been studied and optimized to respect CLIC specifications, fixing the modulator parameters presented in Table 2. Hysteretic controllers have been selected for controlling the switching stages, whereas for the linear stage a PI controller is used. The dimensioning procedure was based on a non-linear optimization approach where all the active bouncer component values (including the number of phases) and the controller parameters were considered as optimization variables. The constraints were the ones presented in Table 1, and the objective function to be minimized was the total active bouncer losses during a complete cycle. In order to reduce the computation time, simplified thermal (switching and conduction losses were evaluated for different 1700V IGBT models) and control (bandwidth, gain and phase margins) design models were used. Results were subsequently validated via numerical simulation. Table 3 presents the active bouncer optimized parameter values for the CLIC specifications.

Table 2 Fixed modulator parameters

\begin{tabular}{|l|c|}
\hline Primary Voltage & $15 \mathrm{kV}$ \\
\hline Main capacitor bank size & $350 \mu \mathrm{F}$ \\
\hline Primary winding leakage inductance & $9 \mu \mathrm{H}$ \\
\hline Primary winding equivalent resistance & $11 \mathrm{~m} \Omega$ \\
\hline Secondary winding leakage inductance & $9 \mu \mathrm{H}$ \\
\hline Secondary winding equivalent resistance & $11 \mathrm{~m} \Omega$ \\
\hline Magnetizing Inductance & $0.122 \mathrm{H}$ \\
\hline
\end{tabular}


Table 3 Optimized Parameters for Bouncer Topologies

\begin{tabular}{|l|c|r|r|}
\hline Topology & Topology \#1 & Topology \#2 & Topology \#3 \\
\hline Number of phases & 4 & $\begin{array}{r}3 \times \text { High Current Stage } \\
4 \times \text { Low Current Stage }\end{array}$ & $\begin{array}{r}3 \times \text { High Current Stage } \\
1 \times \text { Low Current Stage }\end{array}$ \\
\hline $\begin{array}{l}\text { Switching frequency } \\
\text { per phase }\end{array}$ & $62.5 \mathrm{kHz}$ & $\begin{array}{r}26.3 \mathrm{kHz} \text { High Current Stage } \\
110.5 \mathrm{kHz} \text { Low Current Stage }\end{array}$ & $26.3 \mathrm{kHz}$ High Current Stage \\
\hline $\begin{array}{l}\text { Inductance value } \\
\text { per phase }\end{array}$ & $100 \mu \mathrm{H}$ & $\begin{array}{r}1.8 \mathrm{mH} \text { High Current Stage } \\
200 \mu \mathrm{H} \text { Low Current Stage }\end{array}$ & $1.8 \mathrm{mH}$ High Current Stage \\
$1 \mu \mathrm{H}$ Low Current Stage \\
\hline $\mathrm{C}_{\mathrm{b}}$ & $1 \mu \mathrm{F}$ & $1 \mu \mathrm{F}$ & $1 \mu \mathrm{F}$ \\
\hline $\mathrm{R}_{\mathrm{bd}}$ & $0.5 \Omega$ & $0.3 \Omega$ & Not required \\
\hline $\mathrm{C}_{\mathrm{bd}}$ & $4 \mu \mathrm{F}$ & $4 \mu \mathrm{F}$ & Not required \\
\hline
\end{tabular}

Figures 6,7 and 8 show a zoom on the pulse obtained by numerical simulation for topologies 1,2 and 3 , respectively. The optimized topology $\# 1$ consists of a 4 phases buck converter using $1700 \mathrm{~V}, 800 \mathrm{~A}$, IGBTS FZ800R17KF6C_B2 from Infineon. The maximum current and switching frequency per phase are $497 \mathrm{~A}$ and $62.5 \mathrm{kHz}$, respectively. It is necessary to damp the LC output filter with a typical $\mathrm{C}_{\mathrm{bd}}-\mathrm{R}_{\mathrm{bd}}$ parallel damping branch of $\mathrm{C}_{\mathrm{bd}}=4 \mu \mathrm{F}$ and $\mathrm{R}_{\mathrm{bd}}=0.5 \Omega$ to avoid subharmonic oscillations. The performances achieved at the secondary output are a voltage stability of $67 \mathrm{~V}$ and an equivalent frequency ripple of $250 \mathrm{kHz}$.

The optimized topology \#2 is composed of a 3 phases high-current converter using the same $1700 \mathrm{~V}$, 800A, FZ800R17KF6C_B2 IGBTs from Infineon with a maximum current and switching frequency per phase of $644.3 \mathrm{~A}$ and $26.3 \mathrm{kHz}$; and a 4 phases low-current converter using $1700 \mathrm{~V}, 60 \mathrm{~A}$, BSM50GS120DN2 from Infineon, with a maximum current and switching frequency per phase of 19A and $110 \mathrm{kHz}$, respectively. A passive $C_{b d}-R_{b d}$ damping branch is still required with values of $C_{b d}=4 \mu F$ and $\mathrm{R}_{\mathrm{bd}}=0.3 \Omega$. The performances achieved at the secondary output are a voltage stability of $49.39 \mathrm{~V}$ and an equivalent frequency ripple of $442 \mathrm{kHz}$.

The optimized topology \#3 is composed of a 3 phases high-current converter using the same $1700 \mathrm{~V}$, 800A, FZ800R17KF6C B2 IGBTs with a maximum current and switching frequency per phase of $644.3 \mathrm{~A}$ and $26.3 \mathrm{kHz}$; and a low-current linear MOSFET stage with a closed-loop bandwidth of $1 \mathrm{MHz}$ and a maximum output current of $60 \mathrm{~A}$. The damping is performed by the linear stage, so no additional $\mathrm{C}_{\mathrm{bd}}-\mathrm{R}_{\mathrm{bd}}$ passive damping branch is required in this case. The performances achieved at the output are a voltage stability of $44 \mathrm{~V}$ and an equivalent frequency ripple of $71 \mathrm{kHz}$. 

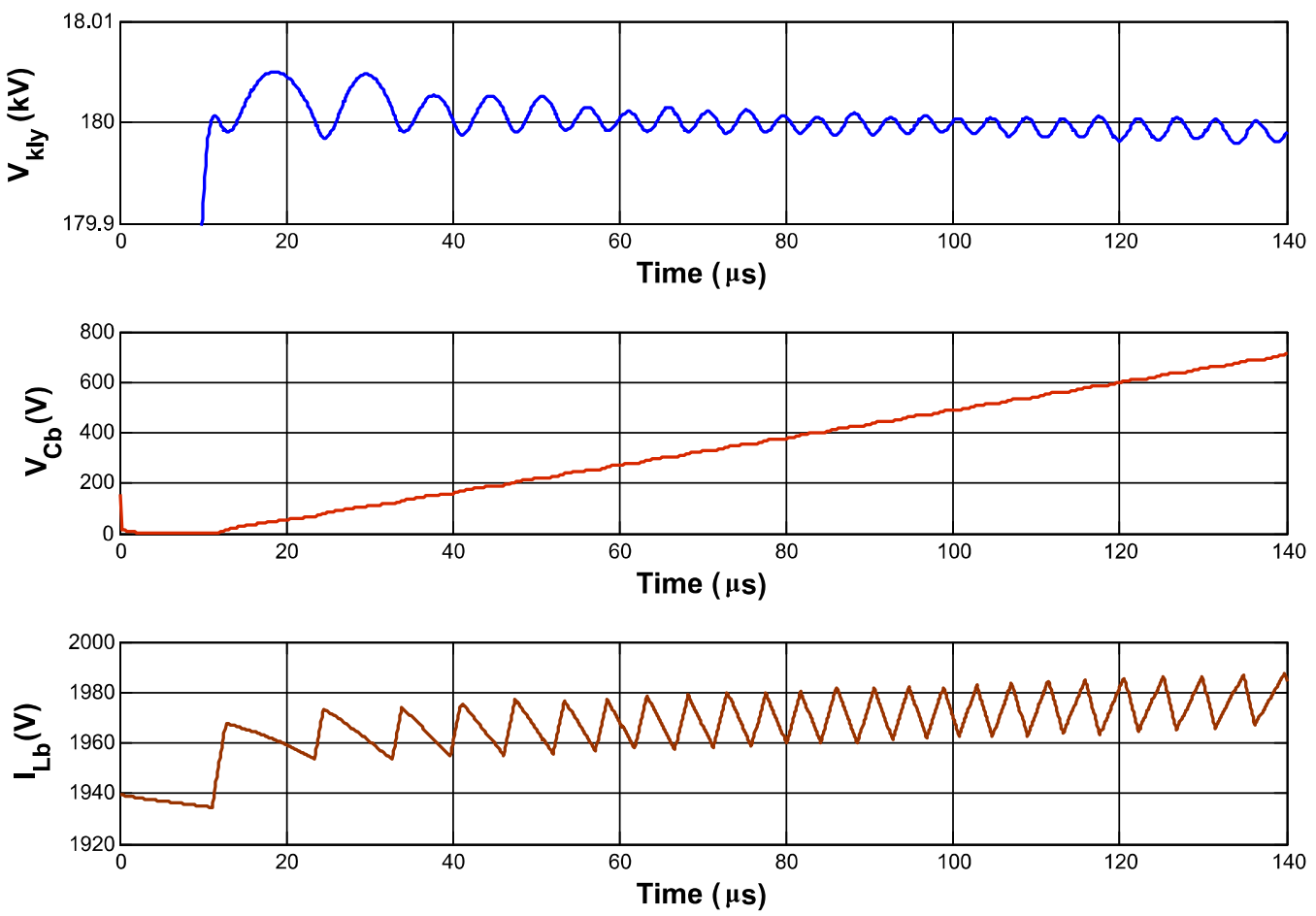

Fig. 6: Topology \#1 numerical simulation results on CLIC specifications.
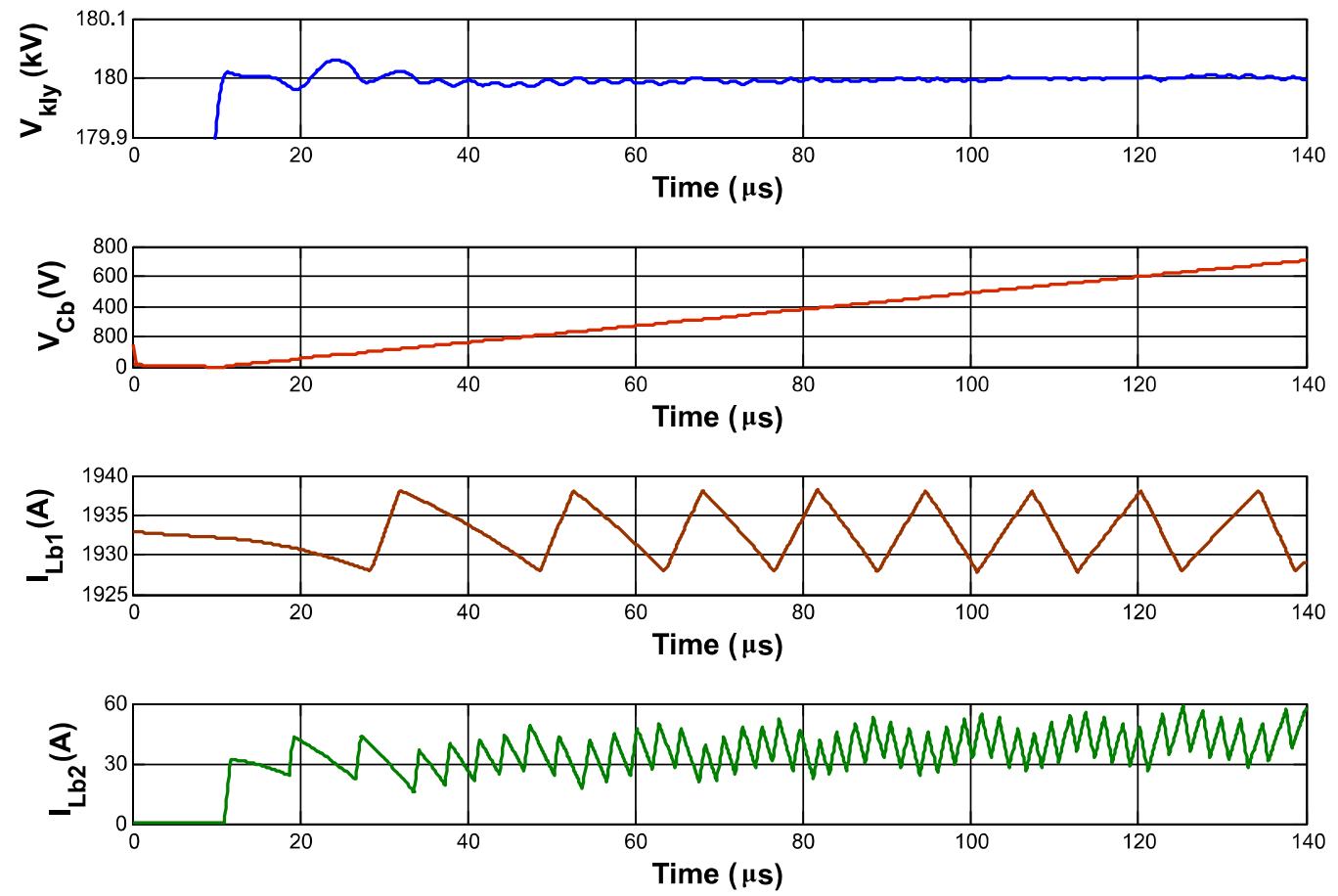

Fig. 7: Topology \#2 numerical simulation results on CLIC specifications. 

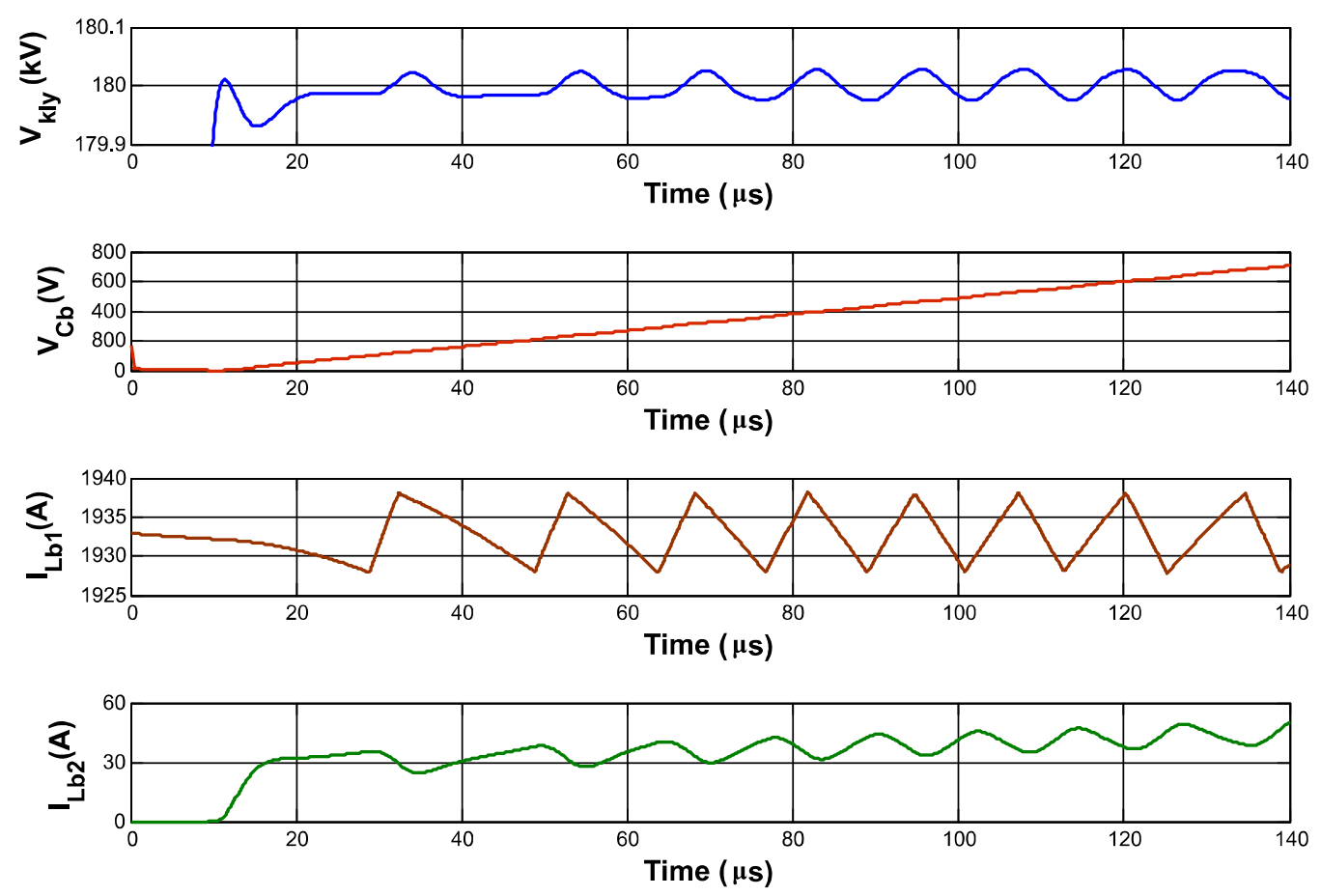

Fig. 8: Topology \#3 numerical simulation results on CLIC specifications.

Table 4 summarizes and compares the characteristics and performances achieved by the different topologies evaluated by means of numerical simulations. We observe how Topologies \#2 and \#3 increase the filter inductance value of the high-current stage to reduce the switching frequency and, therefore, minimize the switching losses present in Topology \#1. Topology \#3 is the one providing the best performances, due to the high bandwidth of the linear converter. Although the average losses during the pulse are higher on Topology \#3 due to the operation of the power MOSFET in its linear region, the lower losses on the bouncer reset (output filter capacitor discharge between pulses) due to the absence of the damping branch (composed of $\mathrm{C}_{\mathrm{bd}}$ and $\mathrm{R}_{\mathrm{bd}}$ ), make this topology also the most efficient. In addition, the lower switching frequency harmonic content at the output makes possible to correct possible repeatability issues on the power system by means of a feedback loop on the low level RF control [9], in order to respect the challenging CLIC repeatability specifications (see Table 1). Therefore, topology \#3 seems to be the most convenient one for the future CLIC klystron modulator active bouncer.

Table 4 Active bouncer topologies comparison

\begin{tabular}{|l|c|c|c|}
\hline & Topology \#1 & Topology \#2 & Topology \#3 \\
\hline $\mathrm{N}^{0}$ of phases High Current Stage & 4 & 3 & 3 \\
\hline $\mathrm{N}^{0}$ of phases Low Current Stage & - & 4 & 1 \\
\hline Av. Losses during the pulse & $985 \mathrm{~W}$ & $446 \mathrm{~W}$ & $490 \mathrm{~W}$ \\
\hline Av. Losses on current rise & $7.3 \mathrm{~W}$ & $43.6 \mathrm{~W}$ & $43.6 \mathrm{~W}$ \\
\hline Av. Losses on current fall & $13 \mathrm{~W}$ & $70 \mathrm{~W}$ & $70 \mathrm{~W}$ \\
\hline Av. Losses on bouncer reset & $110 \mathrm{~W}$ & $110 \mathrm{~W}$ & $30 \mathrm{~W}$ \\
\hline Total average losses & $1.12 \mathrm{~kW}$ & $670 \mathrm{~W}$ & $633 \mathrm{~W}$ \\
\hline Energy recovered through diode $\mathrm{D}_{\mathrm{f}}$ & $0.20 \mathrm{~J}$ & $55 \mathrm{~J}$ & $55.5 \mathrm{~J}$ \\
\hline Passive damping required? & Yes & Yes & $\mathrm{No}$ \\
\hline Output ripple frequency & $250 \mathrm{kHz}$ & $442 \mathrm{kHz}$ & $71 \mathrm{kHz}$ \\
\hline
\end{tabular}




\begin{tabular}{|l|c|c|c|}
\hline Total $\mathrm{LI}^{2} / 2$ & $193.45 \mathrm{~J}$ & $1.12 \mathrm{~kJ}$ & $1.2 \mathrm{~kJ}$ \\
\hline Capacitor bank size & $54 \mathrm{mF}(1 \mathrm{kV})$ & $60 \mathrm{mF}(1 \mathrm{kV})$ & $60 \mathrm{mF}(1 \mathrm{kV})$ \\
\hline Output stability at $180 \mathrm{kV}$ & $67 \mathrm{~V}$ & $50 \mathrm{~V}$ & $44 \mathrm{~V}$ \\
\hline Redundancy modules required & 1 module & 2 modules & 2 modules \\
\hline Control \& Electronics complexity & Medium & Medium & Low \\
\hline Main challenges/difficulties & Control Loop & Control+HF stage & MOSFET stage \\
\hline
\end{tabular}

\section{Conclusion}

The proposed active bouncer topologies are able to comply with very fast rise time and very repeatable klystron modulators. The solution which combines a high current - low switching frequency stage with a low current - fast switching one, reveals better performances than a classical multiphase interleaved power converter active bouncer. Indeed the majority of the active bouncer current is managed by a much higher efficient power converter, and the fine voltage droop regulation is performed via a highly dynamical, and small sized, power converter. Topology \#3 achieves better performances than topology \#2 due to the higher bandwidth of the linear stage, and higher efficiency due to the absence of a parallel damping branch in its output filter which must be discharged between two consecutive pulses. The lower switching frequency harmonic content of Topology \#3 makes also possible to compensate possible repeatability errors by means of a feedback loop on the low level RF control, in order to respect the challenging repeatability specifications of CLIC. Therefore, for the specific case of CLIC application, topology \#3 would be the best solution.

\section{References}

[1] Gribble R.F., Lynch M.T., Rees D.E., Tallerico P.J., Reass W.A., Doss J.D. and Borovina D.L.: Operational results of the spallation neutron source (SNS) polyphase converter-modulator for the $140 \mathrm{kV}$ klystron RF system, Proceedings of the 2001 Particle Accelerator Conference, Chicago, 2001.

[2] Kemp M. A., Benwell A., Burkhart C., Larsen R., MacNair D., Nguyen M., Olsen J.: Final Design of the SLAC P2 Marx Klystron Modulator, SLAC-PUB-14537.

[3] Cortazar O. D., Ganuza D., De la Fuente J. M., Zulaika M., Pérez A., Anderson D. E.: A 100 kV, 60 A solid state $4 \mathrm{kHz}$ switching modulator for high power klystron driving, Review of Scientific Instruments, 84, 2013.

[4] Mulvaney J.M., Kempkes M.A, Gaudreau M.P.J., Casey J.: Solid-State High Voltage Pulse Modulators for High Power Microwave Applications, Proceedings of EPAC, Vienna, Austria, 2000.

[5] Iten M., Reimann D., Troxler J., Gensch U., Grimberg M., Jachmann L., Koehler W., Leich H., Penno M., Wenndor R., Choroba S., Eckoldt H. J., Grevsmuehl T., Alex J., Bader M.: A new prototype modulator for the European XFEL project in pulse step modulator technology, Proceedings of PAC09, Vancouver, BC, Canada, 2009.

[6] Aguglia D.: 2MW active bouncer converter design for long pulse klystron modulators, Proceedings of the $14^{\text {th }}$ European Conference on Power Electronics and Applications, September 2011, Birmingham.

[7] Cabaleiro Magallanes F., Aguglia D., Viarouge P., de Almeida Martins C., Cros J.: A novel active bouncer system for klystron modulators with constant AC power consumption, IEEE Pulsed Power Conference, June 2013, San Francisco.

[8] Aguglia D., de Almeida Martins C., Cerqueira Bastos M., Nisbet D., Siemaszko D., Sklavounou E., Viarouge P.: Klystron Modulator Technology Challenges for the Compact Linear Collider (CLIC), IEEE Pulsed Power Conference, June 2011.

[9] Dal Gobbo A. and Aguglia D.: Global Design Analysis for Highly Repeatable Solid-state klystron Modulators, IEEE HVPM Conference, Santa Fe (NM), pp. 1-4, 2014. 